\title{
The association between expression of IncRNAs in patients with GDM
}

\author{
Li Yuanyuan ${ }^{1}$, Li Dongmei ${ }^{1}$ and Cheng Xingbo ${ }^{2}$ \\ ${ }^{1}$ Department of Endocrinology, Inner Mongolia People's Hospital, Hohhot, Inner Mongolia, China \\ ${ }^{2}$ Department of Endocrinology, The First Affiliated Hospital of Soochow University, Suzhou, China \\ Correspondence should be addressed to C Xingbo: hewsmzyh@icloud.com
}

\begin{abstract}
Objective: Gestational diabetes mellitus (GDM) is common worldwide and seriously threatens maternal and infant health. The expression of non-coding (ncRNA) is tissuespecific and highly stable in eukaryotic cells and the circulatory system, which can act as an early molecular marker of GDM.

Methods: The differential expression of IncRNA and mRNA in the peripheral blood of patients with GDM (experimental group) and healthy pregnant women (control group) was analysed via IncRNA gene chip. Employing biological function clustering and KEGG signalling pathway analysis, we selected the mRNAs and IncRNAs closely related to the insulin signalling pathway of GDM to analyse the possible regulatory mechanism in the pathogenesis of GDM. The sequencing results were further verified via quantitative PCR (Q-PCR).

Results: LncRNA microarray analysis revealed 7498 genes (3592 upregulated,

3906 downregulated) differentially expressed in the GDM group and healthy pregnant women control group, including 1098 differentially expressed IncRNAs (609 upregulated, 489 downregulated). According to the regulatory pathway of the IncRNA mRNA network, 6 IncRNAs and 4 mRNAs were found to play a significant role in insulin resistance. Conclusions: The IncRNAs ERMP1, TSPAN32 and MRPL38 form a co-expression network with TPH1, which is mainly involved in the tryptophan metabolism pathway and in the development of GDM. Moreover, IncRNA RPL13P5 forms a co-expression network with the TSC2 gene via the PI3K-AKT and insulin signalling pathways, which are involved in the process of insulin resistance in GDM.
\end{abstract}

\author{
Key Words \\ - gestational diabetes \\ mellitus \\ - IncRNA \\ - tryptophan metabolism \\ pathway \\ - PI3K-AKT \\ - insulin signalling pathways
}

\section{Introduction}

The burgeoning epidemic of gestational diabetes mellitus (GDM) threatens maternal and infant health. GDM is characterized by glucose intolerance, which causes poorly controlled diabetes during pregnancy. Insulin resistance is an early determinant of declining $\beta$-cell function (1); however, the differential expression of genes underlying these phenomena is not fully understood. Differences in genetic background likely explain these differences in gene expression $(2,3)$. After a critical point, insufficient insulin secretion and increased insulin requirements could lead to consequent hyperglycaemia in GDM (4). Although routine examination (via fasting or postprandial blood glucose) can be fast-evolving, this is rarely effective in making an accurate and timely diagnosis. A combination of one or more molecular markers is urgently needed to monitor insulin resistance in its early stages (5).

During pregnancy, glucose is a primary source of foetal energy. As the pregnancy progresses, a foetus' need for glucose gradually increases. Therefore, this increase in maternal glucose consumption puts the mother in a state

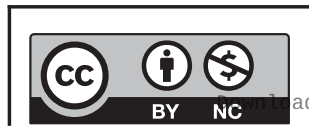

This work is licensed under a Creative Commons Attribution-NonCommercial 4.0 International License. ded from Bioscientifica.com at 04/26/2023 10:37:52AM 
of 'accelerated hunger'. Regulatory mechanisms, such as lipodieresis and glyconeogenesis, are needed by the mother to elevate foetal blood sugar during hypoalimentation or a reduction in blood sugar. During the first trimester of pregnancy, only slight changes in blood sugar and insulin sensitivity are observed, whereas insulin secretion is higher and anabolic processes are prioritized, allowing for the storage of more fat and energy. In late pregnancy, the i.v. glucose tolerance test shows that insulin in the first and second secretory phases increases three-fold after stimulation possibly to compensate for insulin resistance and decreased sensitivity.

In DNA-templated organic synthesis, only $2 \%$ of the genome is transcribed into proteins, whereas the remaining 98\% are called ncRNA (6). Conversely, lncRNAs are a collection of long noncoding exons (>200 nt). In the past, very few tools were available for large-scale sequencing of lncRNA (7). Thus, lncRNA was once seen as irrelevant 'transcription noise,' but it has since been widely implicated in regulating many of the genes that are responsible for metabolic processes. It does so by adjusting related protein-coding genes through a variety of ways at different levels; lncRNA and DNA bases can be inserted within three base pairs, thus influencing the expression of target genes (8).

Information on molecules that regulate gene expression allows for gene expression ranking. These molecules are ubiquitous at the epigenetic, transcriptional and post-transcriptional processes. For example, lncRNAs take part in almost all physiological and pathophysiological processes in an organism $(9,10,11)$; they exhibit a tissue-specific expression and are stable when expressed in eukaryotic cells. Thus, they can be used as an early molecular marker of GDM.

The expression of lncRNA is stable in the blood (12), urine (13) and other body fluids and is even resistant to digestion by RNA enzymes (14). This characteristic allows it to be a non-invasive disease marker. Many lncRNAs with low expression levels are mainly located in the nucleus, and their sequence conservation has high specificity. Recent reports on lncRNAs associated with local gene regulation further support this view. In many cases, it is suggested that the regulatory locus controlling the expression of transcription or the DNA elements within the lncRNA have more activity (15). However, we found very few studies that have identified an association between insulin resistance in GDM and lncRNAs via high-throughput methods (i.e. microarray and RNA-seq).

In this study, lncRNA is monitored at the early stage of insulin resistance in GDM to explore the effects of the changes in susceptibility genes for diabetes and their expression. In our study, elevated glucose levels of oral glucose tolerance test (OGTT) were used as a diagnostic procedure for GDM. Plasma samples from women with and without GDM were collected, and a global genome microarray analysis revealed differentially expressed IncRNAs. In addition, a functional analysis of the altered molecular pathways was conducted. The potential functions of differentially expressed IncRNAs can be predicted.

\section{Materials and methods}

\section{Study population}

This research flow chart is summarized in Fig. 1. Casecontrol studies were conducted at the Inner Mongolia Autonomous Region People's Hospital, Hohhot, China, from 8 October 2019 to 15 March 2020. Plasma samples were obtained from pregnant women both with and without GDM at 24-40 weeks, We measured the BMI, fasting plasma glucose (FPG) level, $1 \mathrm{~h}$ glucose load, $2 \mathrm{~h}$ glucose load, fasting insulin level, c-peptide level and glycosylated haemoglobin level. In addition, a homeostatic model assessment for insulin resistance (HOMA-IR) was conducted. GDM was diagnosed according to the 2019 American Diabetes Association (ADA) criteria (16). All patients underwent an oral $75 \mathrm{~g}$ glucose tolerance test at 24-28 weeks with overnight fasting for $10 \mathrm{~h}$. Plasma glucose test measurement was performed at 1 and $2 \mathrm{~h}$ after oral $75 \mathrm{~g}$ glucose tolerance. A GDM diagnosis was made when the plasma glucose values exceeded any of the defined thresholds (fasting: $92 \mathrm{mg} / \mathrm{dL}(5.1 \mathrm{mmol} / \mathrm{L}) ; 1 \mathrm{~h}: 180 \mathrm{mg} / \mathrm{dL}$ $(10.0 \mathrm{mmol} / \mathrm{L})$; and $2 \mathrm{~h}: 153 \mathrm{mg} / \mathrm{dL}(8.5 \mathrm{mmol} / \mathrm{L})$ ). Patients with complications of diabetes mellitus, chronic hypertension, multiple pregnancies, pre-eclampsia, obesity (BMI $\geq 30$ (1)), and inflammatory diseases were excluded. This study was approved by the Institutional Ethics Committee of the Inner Mongolia Autonomous Region People's Hospital.

\section{Microarray data, screening and functional analysis of differentially expressed genes}

The transcriptome profiles were selected from the venous plasma sample ( $n=6$, three from GDM and three from control individuals). Venous vacuum blood collection (whole blood RNA tube, PAXgene blood collection) was performed to extract $2 \mathrm{~mL}$ of peripheral blood from the

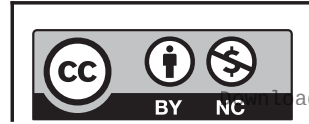

This work is licensed under a Creative Commons Attribution-NonCommercial 4.0 International License. ded from Bioscientifica.com at 04/26/2023 10:37:52AM 


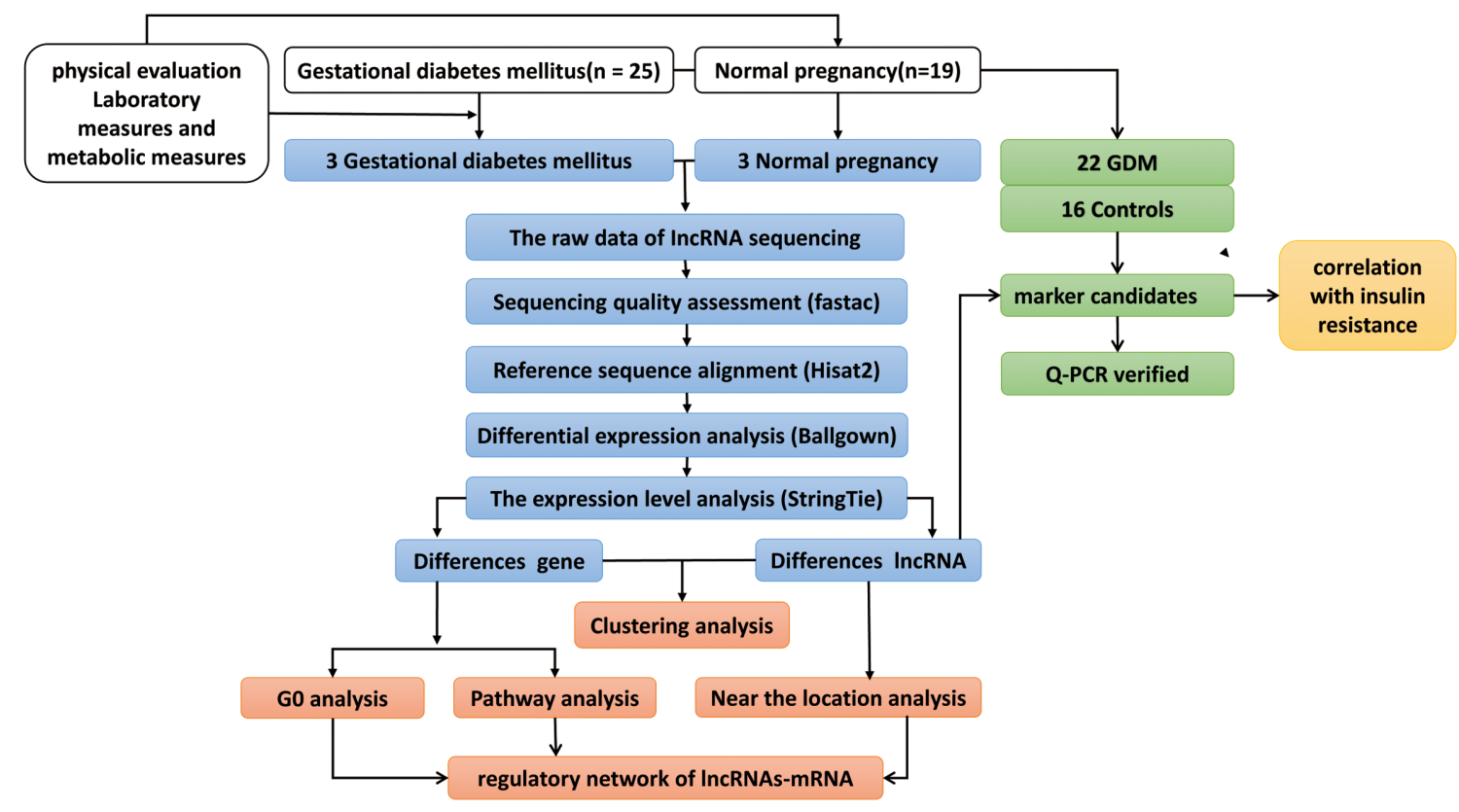

Figure 1

Research flow chart.

patients. The total RNA from each sample was subjected to the NEB\# E7335L, NEB\# E6310L and NEB\# E7760L Ultra Directional RNA Library Prep Kit (NEB, USA). Welch's $t$-test was adopted for the analysis of microarray data and the identification of statistical specifications for significance. The differential expression of lncRNA and mRNA was screened for greater changes. A $P$-value of $\leq 0.05$ was considered statistically significant. The sequences of the clustered transcriptome assembly were compared with public databases. Gene ontology (GO) analysis was conducted for differentially expressed genes. The signalling pathways of these proteins and the functional categories of the unigenes were analysed using the KEGG database. The overall design of the four-plex experiments is illustrated in Fig. 1. Details of the procedures can be found in the supplementary materials.

\section{Construction of mRNA-IncRNA co-expression network}

The mRNA-lncRNA co-expression network was constructed based on the result of Pearson's correlation analysis conducted on the differential expressions of lncRNAs and mRNAs. The mRNA-lncRNA pairs with a significant correlation coefficient were selected. The programme plots the fraction of the edges in a network graph with connections (edges) between all nodes in the network. The stronger the association of neighbouring genes or lncRNAs with a gene, the higher the degree and the more important the status.

A pathway interaction network named Path-Net is constructed based on interaction relationships between pathways in the KEGG database. The degree of the pathway is used as a criterion for assessing the pathway in Path-Net. 'Degree' refers to the number of relationships between a node on the network and surrounding nodes. The larger the degree, the more pathways that interact with it.

Pearson correlation analysis is carried out based on the expression differences of lncRNA and mRNA to construct an mRNA-lncRNA co-expression network. The differences in the co-expression network are used to analyse differences in expression regulatory mechanisms of these mRNA/lncRNA and identify the core positions of this mRNA/lncRNA in the co-expression network. In addition, the functions of surrounding mRNAs in the co-expression network are used to predict the function of unknown lncRNAs. mRNAlncRNA pairs with significant correlation coefficient are selected and the expression correlation between mRNA and lncRNA is used to construct the co-expression network.

\section{RNA extraction and quantitative polymerase chain reaction (Q-PCR)}

Q-PCR was adopted to verify the microarray results in the GDM group $(n=3)$ vs control group $(n=3)$ with gene-specific primers. Total RNA was removed from the

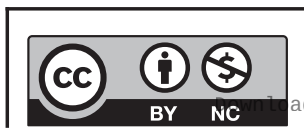

This work is licensed under a Creative Commons Attribution-NonCommercial 4.0 International License. ded from Bioscientifica.com at 04/26/2023 10:37:52AM 
serum that had been stored at $-80^{\circ} \mathrm{C}$ using Trizol. The differentially expressed 1 cRNAs and mRNAs were validated in an independent cohort. Q-PCR was performed using the ChamQTM SYBR qPCR Master Mix (vazyme Q311-02) and monitored using a GeneAmp PCR System 9700. The lncRNA with the best diagnostic value was selected as the biomarker.

\section{Statistical analysis of IncRNAs}

Data analysis was conducted using the SPSS software (version 18.0) (SPSS). All data were expressed as mean \pm S.D. The expression level of the lncRNAs was calculated using the following formula: $\Delta \Delta \mathrm{CT}=\Delta \mathrm{CT}$ (target gene) $-\Delta \mathrm{CT}$ (internal reference gene), where $\Delta \mathrm{CT}$ is the cycle number at which the fluorescence signal crosses the threshold, and $\Delta \Delta \mathrm{CT}$ is a simplified form of the relative fluorescence quantitative calculation formula that compares the difference or ratio between the different samples. $P$-values of $<0.05$ were considered statistically significant.

\section{Results}

\section{Maternal characteristics}

A total of 44 cases were included in this study (25 patients with GDM and 19 healthy controls). The two pairs of peripheral blood samples were aged-matched. Prepregnancy BMI was calculated based on the self-reported weight and height before pregnancy, whereas FPG was examined on gestational week 36. No significant differences were observed between the GDM group and the control group. The sample for this data is outlined in Table 1.

\section{Quality of RNA data output}

The general data quality requirements for high-traffic sorting are as follows: Q30 > 85\% indicates satisfactory data quality, with higher Q scores associated with a lower probability of error. The lncRNA and mRNA data of this sequencing were greater than $90 \%$, and the error rate of base sequencing was less than $0.02 \%$. The data quality is sufficient for subsequent analysis. All available ships were included in the analysis.

\section{Construction of differential expression profiles of IncRNA and mRNA in the peripheral blood of patients with GDM}

To study the differences between the three control subjects and three patients with GDM, the two groups
Table 1 Characteristics of the study participants.

\begin{tabular}{|c|c|c|c|}
\hline Characteristics & $\begin{array}{c}\text { GDM } \\
(n=25)\end{array}$ & $\begin{array}{c}\text { Control group } \\
\qquad(n=19)\end{array}$ & P-value \\
\hline Age, years & $31.68 \pm 0.7499$ & $30.16 \pm 0.8661$ & 0.1907 \\
\hline Pre-pregnancy BMI & $23.79 \pm 0.6881$ & $21.49 \pm 0.7277$ & 0.0287 \\
\hline FBG (mmol/L) & $5.739 \pm 0.3522$ & $4.286 \pm 0.0531$ & 0.0009 \\
\hline $1 \mathrm{~h}$ & $10.82 \pm 0.7044$ & $7.154 \pm 0.3189$ & 0.0001 \\
\hline $2 \mathrm{~h}$ & $9.264 \pm 0.4022$ & $6.079 \pm 0.2557$ & $<0.0001$ \\
\hline INS (mU/L) & $11.41 \pm 1.129$ & $6.552 \pm 0.5757$ & 0.0012 \\
\hline HOMA-IR & $2.62 \pm 0.2714$ & $1.246 \pm 0.1083$ & 0.0001 \\
\hline
\end{tabular}

were compared, and differential gene screening was performed to methodically investigate lncRNAs in GDM. Transcriptome analysis was conducted to display the expression outlines of lncRNAs. To screen the differentially expressed genes, $\mid \log 2$ fold change $\mid>1.2$ and $P<0.05$ were used. The consequences of the microarray analysis established evident differences in the expression profiles of lncRNAs and mRNAs between patients with GDM and the control subjects. A total of 7498 differentially expressed mRNAs (3592 upregulated and 3906 downregulated) and 1098 differentially expressed lncRNAs (609 upregulated and 489 downregulated) were found in this sequencing (Table 2). The cluster heatmap revealed the differential expression of two kinds of RNAs in the samples of the three control subjects and three patients with GDM. To specify the genes observed via differential screening, we conducted cluster analysis depending on the signal value of each gene in the sample (Fig. 2A, B, C and D).

In Fig. $2 \mathrm{~A}$ and $\mathrm{B}$, the genes identified on differential screening underwent clustering analysis according to the signal value of each gene in the samples. The abscissa represents the sample names between the groups, whereas the ordinate represents the differentially expressed genes. Red indicates the high expression of differentially expressed genes, whereas green indicates the minimal expression of differentially expressed genes in the samples.

In Fig. 2C and D, upregulated genes with different multiples greater than 1.2 and $P$-values of $\leq 0.05$ are indicated by red dots. Those with a unique multiple less than 0.83333 and a $P$-value of $\leq 0.05$ are downregulated genes, which are indicated in green. The genes that were not significantly different are indicated by grey dots.

The differences were examined based on a $P$-value of $<0.05$ and an absolute difference of $+/-$ two-fold (i.e. log change $>+/-2.0$ ). A total of 3971 lncRNA-mRNA pairs with significant correlations in expression were screened. The functions of IncRNA were determined from the known functions of mRNA; functional enrichment analysis was conducted for significantly correlated mRNAs with 
Table 2 Top differentially expressed mRNAs and IncRNAs.

\begin{tabular}{|c|c|c|}
\hline Gene symbol & Length & Log2 fold change \\
\hline \multicolumn{3}{|l|}{ IncRNA } \\
\hline AL732372.2 & 9944 & 24.33780372 \\
\hline FAS & 12,963 & 24.29753701 \\
\hline SUMO3 & 13,163 & 21.936314 \\
\hline NOP53 & 11,180 & 21.66537943 \\
\hline LINC00894 & 5682 & 21.23578621 \\
\hline CDK19 & 1967 & 21.23578621 \\
\hline AC092821.3 & 22,711 & 21.23578621 \\
\hline POLR1D & 17,202 & 21.22407648 \\
\hline ERCC6 & 43,335 & 20.89981794 \\
\hline CBWD3 & 46,292 & 20.83406406 \\
\hline CDKN3 & 18,489 & -22.089849 \\
\hline FAM102A & 9840 & -22.09430301 \\
\hline AL732372.2 & 15,665 & -22.10367387 \\
\hline ZMYM1 & 36,464 & -22.19318039 \\
\hline $\mathrm{BRI3}$ & 3920 & -22.21577988 \\
\hline THUMPD3-AS1 & 8613 & -22.40982355 \\
\hline LRRC75A-AS1 & 2910 & -22.76180551 \\
\hline $\operatorname{cox} 20$ & 9399 & -22.96916569 \\
\hline CEP162 & 52,837 & -23.38720014 \\
\hline DNAJC6 & 117,976 & -25.08352346 \\
\hline \multicolumn{3}{|l|}{ mRNA } \\
\hline ASAP1 & 349,127 & 27.29460959 \\
\hline SERF2 & 17,496 & 26.83679243 \\
\hline IFITM1 & 1714 & 26.52378758 \\
\hline HNRNPU & 9448 & 26.14058329 \\
\hline DIAPH1 & 104,034 & 25.80045113 \\
\hline IKZF1 & 100,317 & 25.63427718 \\
\hline BNIP3L & 27,801 & 25.55028294 \\
\hline TRRAP & 132,150 & 25.53453387 \\
\hline SPG11 & 100,822 & 25.18641404 \\
\hline FLNA & 26,107 & 24.84383539 \\
\hline CYFIP2 & 127,085 & -25.58554936 \\
\hline NKTR & 48,048 & -25.86487733 \\
\hline SERP1 & 61,177 & -25.90150098 \\
\hline EIF4E3 & 49,828 & -25.91873442 \\
\hline RPS3A & 4967 & -26.03324779 \\
\hline HNRNPUL1 & 41,898 & -26.0500817 \\
\hline SPOCK2 & 29,998 & -26.39218862 \\
\hline KMT2E & 99,895 & -27.53008692 \\
\hline RPS27A & 3686 & -27.54330358 \\
\hline SNORD3A & 217 & -29.60661663 \\
\hline
\end{tabular}

\begin{tabular}{l}
\hline P-value \\
\hline \\
$4.68174 \mathrm{E}-10$ \\
$1.45924 \mathrm{E}-11$ \\
$1.98532 \mathrm{E}-08$ \\
$2.96336 \mathrm{E}-08$ \\
$5.53507 \mathrm{E}-08$ \\
$5.53507 \mathrm{E}-08$ \\
$5.53507 \mathrm{E}-08$ \\
$5.63745 \mathrm{E}-08$ \\
$8.978 \mathrm{E}-08$ \\
$9.85969 \mathrm{E}-08$ \\
$1.59153 \mathrm{E}-08$ \\
$1.58093 \mathrm{E}-08$ \\
$1.55884 \mathrm{E}-08$ \\
$1.36248 \mathrm{E}-08$ \\
$1.31681 \mathrm{E}-08$ \\
$9.81644 \mathrm{E}-09$ \\
$5.70527 \mathrm{E}-09$ \\
$4.15816 \mathrm{E}-09$ \\
$2.16163 \mathrm{E}-09$ \\
$1.36117 \mathrm{E}-10$ \\
\\
$2.819 \mathrm{E}-12$ \\
$4.46397 \mathrm{E}-16$ \\
$1.12811 \mathrm{E}-11$ \\
$2.21623 \mathrm{E}-11$ \\
$7.14906 \mathrm{E}-15$ \\
$5.33118 \mathrm{E}-11$ \\
$6.1571 \mathrm{E}-11$ \\
$6.32523 \mathrm{E}-11$ \\
$1.14317 \mathrm{E}-10$ \\
$2.03059 \mathrm{E}-10$ \\
$5.79726 \mathrm{E}-11$ \\
$3.58405 \mathrm{E}-11$ \\
$3.36355 \mathrm{E}-11$ \\
$3.26444 \mathrm{E}-11$ \\
$2.67472 \mathrm{E}-11$ \\
$9.36737 \mathrm{E}-16$ \\
$1.42471 \mathrm{E}-11$ \\
$1.832 \mathrm{E}-12$ \\
$1.788 \mathrm{E}-12$ \\
$4.10063 \mathrm{E}-19$ \\
\end{tabular}

\section{padj}

$4.95613 \mathrm{E}-07$

$2.84816 \mathrm{E}-08$

$5.85696 \mathrm{E}-06$

7.4481E-06

$1.16621 \mathrm{E}-05$

$1.16621 \mathrm{E}-05$

$1.16621 \mathrm{E}-05$

$1.18354 \mathrm{E}-05$

$1.75508 \mathrm{E}-05$

1.88323E-05

$5.07163 \mathrm{E}-06$

5.07163E-06

5.04468E-06

$4.73528 \mathrm{E}-06$

4.67303E-06

3.82003E-06

$2.79482 \mathrm{E}-06$

2.361E-06

$1.55185 \mathrm{E}-06$

$1.7529 \mathrm{E}-07$

7.92548E-09

$3.48513 \mathrm{E}-12$

2.42964E-08

$4.13198 \mathrm{E}-08$

4.05923E-11

7.92797E-08

8.84047E-08

8.97866E-08

$1.50316 \mathrm{E}-07$

2.4868E-07

8.51965E-08

5.94006E-08

$5.722 \mathrm{E}-08$

5.66362E-08

4.84226E-08

$6.15861 \mathrm{E}-12$

$2.84816 \mathrm{E}-08$

5.44873E-09

5.44756E-09

5.12235E-15
Style

Up

Up

Up

Up

Up

Up

Up

Up

Up

Up

Down

Down

Down

Down

Down

Down

Down

Down

Down

Down

Up

Up

Up

Up

Up

Up

Up

Up

Up

Up

Down

Down

Down

Down

Down

Down

Down

Down

Down

Down differentially expressed lncRNA. Three GO terms, biological process (BP), cellular component (CC) and molecular function (MF), were utilized for the analysis using medium stringency settings. Biological process (BP) included 369 genes downregulated and 332 genes upregulated by BP, 90 downregulated and 65 upregulated by CC and 62 downregulated and 81 upregulated by MF (Fig. 3A and B). The top GO explanations were largely distributed amongst the MF, CC and BP subscriptions. Amongst the lncRNAmRNA genes differentially sequenced, the top 10 had their expression upregulated and downregulated by BP. The functions related to selected genes are presented in Fig. 3C and D.

To create the KEGG database, Fisher's exact test and chisquared test were adopted to conduct pathway analysis. We analysed the significance of the pathway involved in the target genes. Pathway enrichment analyses were conducted based on the KEGG pathway analysis. Pathways with more explanations than anticipated with the differentially expressed genes $(P<0.05)$ are emphasized in Fig. $3 \mathrm{E}$ and F. Built on the KEGG analysis, 15 downregulated and 18 upregulated genes were screened. https://ec.bioscientifica.com

https://doi.org/10.1530/EC-21-0227 (c) 2021 The authors Published by Bioscientifica Ltd

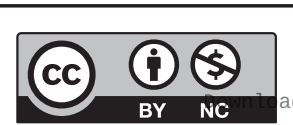

This work is licensed under a Creative Commons Attribution-NonCommercial 4.0 International License. ded from Bioscientifica.com at 04/26/2023 10:37:52AM via free access 
A

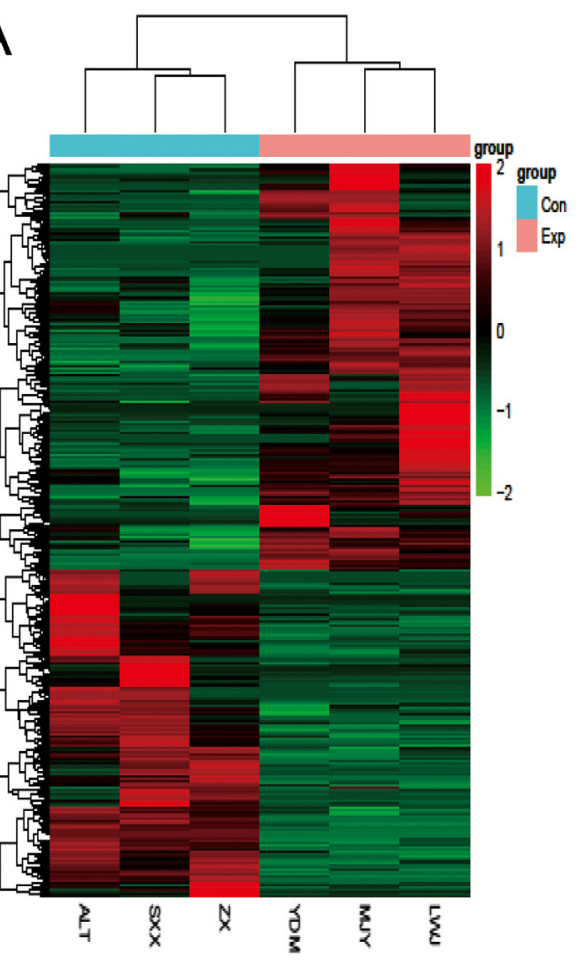

B

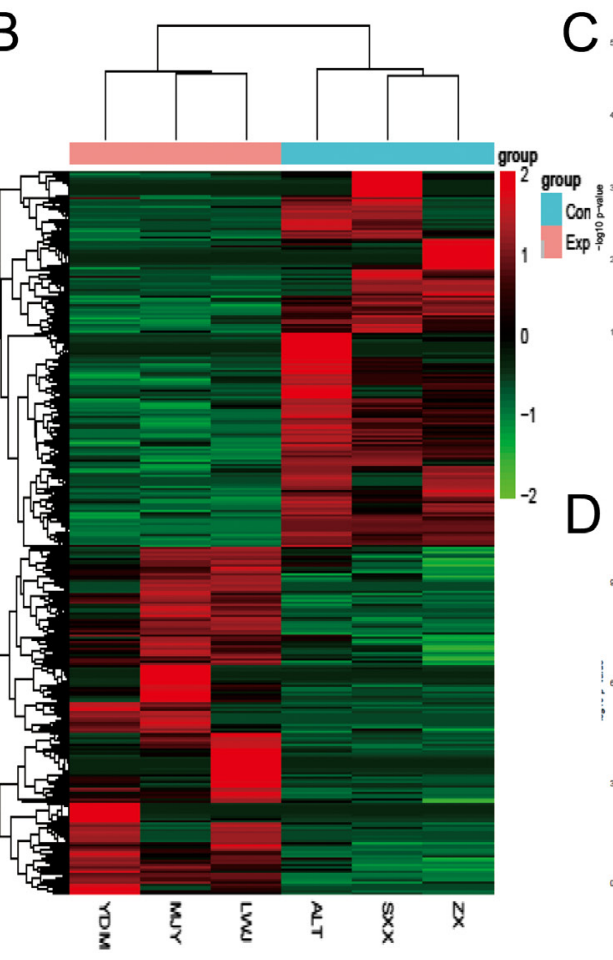

C.

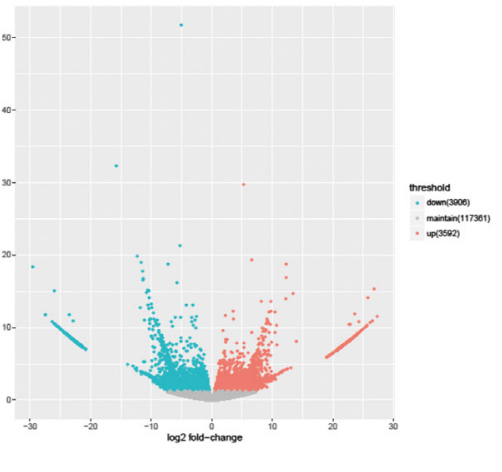

D

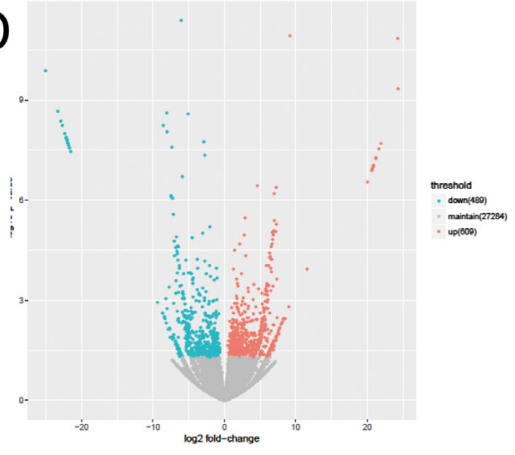

Figure 2

Heatmap and volcano analyses of differentially expressed IncRNAs and mRNAs.

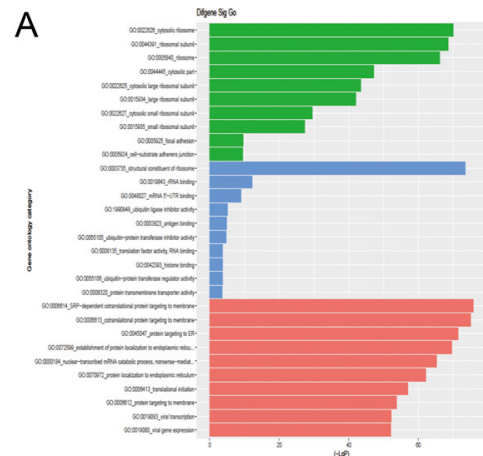

B

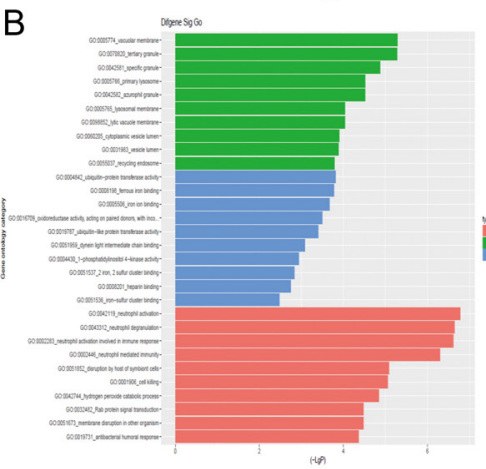

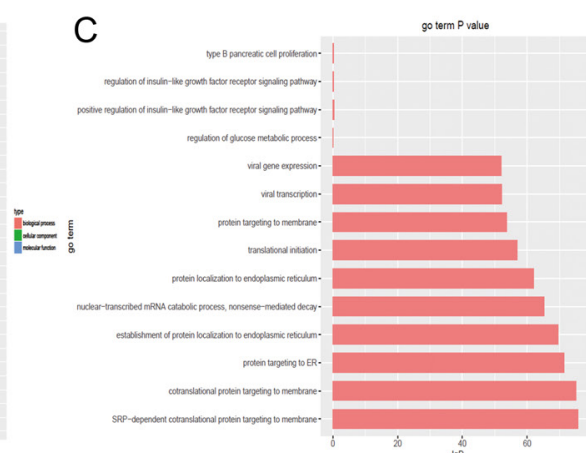

$\mathrm{D}$

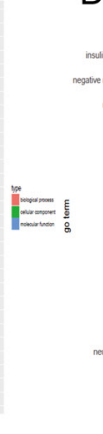

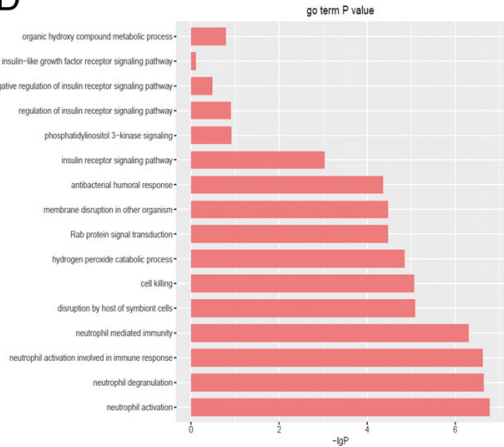

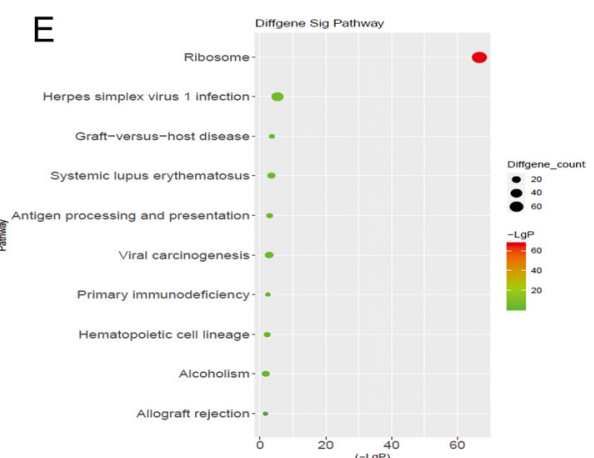

$\mathrm{F}$

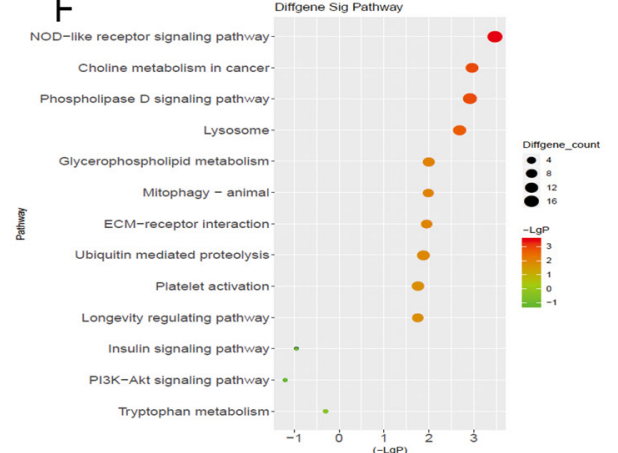

Figure 3

Microarray data, screening and functional analysis of differentially expressed genes.

https://ec.bioscientifica.com

https://doi.org/10.1530/EC-21-0227
() 2021 The authors Published by Bioscientifica Ltd
(C) $(1) \Theta$ Attribution-NonCommercial 4.0 International License. ded from Bioscientifica.com at 04/26/2023 10:37:52AM 


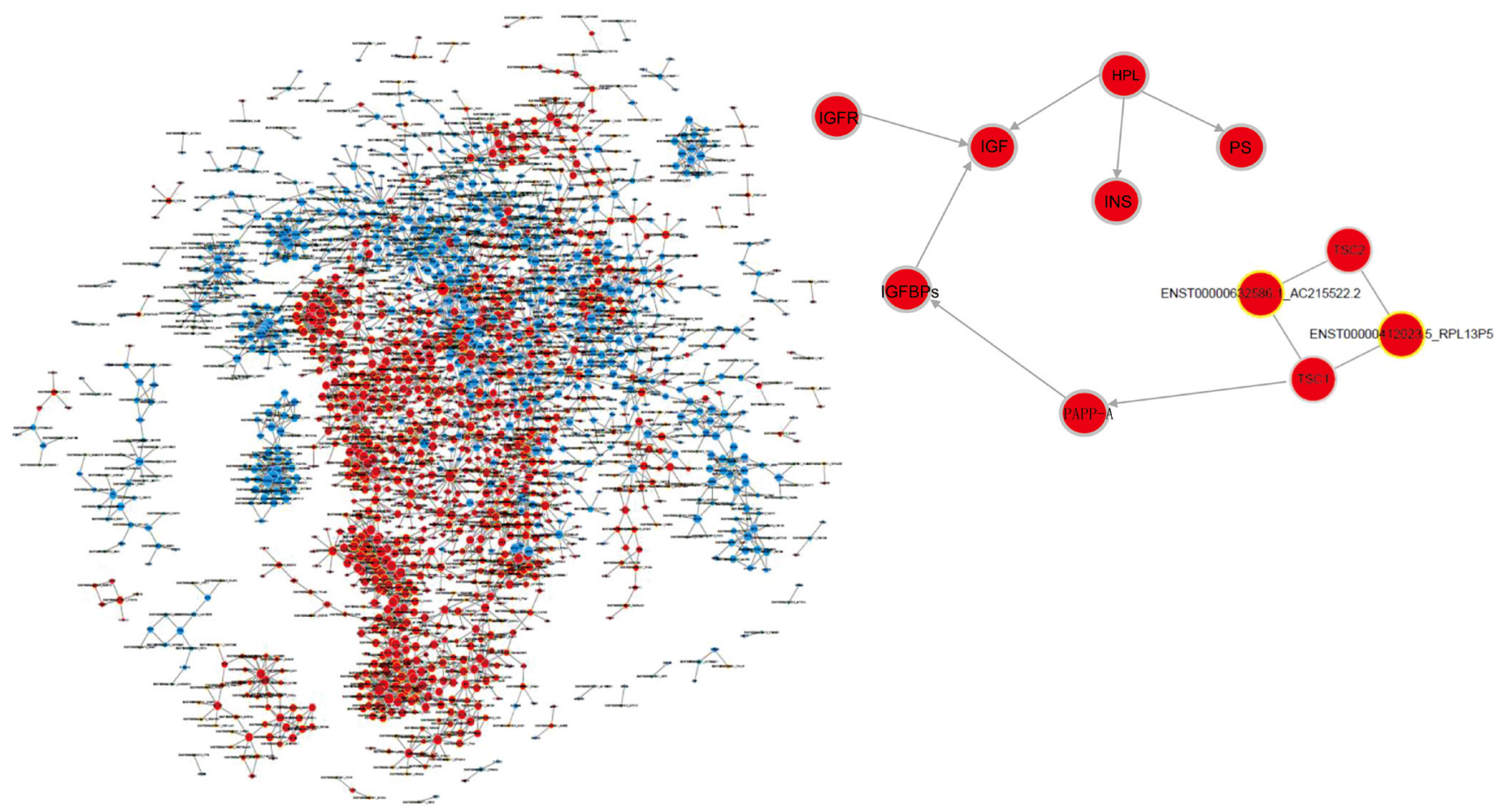

Figure 4

The mRNA-IncRNA co-expression network.

\section{The mRNA-IncRNA interaction network}

The expressions of mRNA and lncRNA create differences between the lncRNA and mRNA express networks. The differences in the expressions reflect the differences in the regulatory mechanism of gene expression, pinpoint the mRNA/lncRNAs expressed in total in the core position on the network and allow the total peripheral mRNA express network to predict unknown lncRNA functions (Fig. 4). In the network, global and systematic pathway analysis can be carried out on the signal transduction relationships between significant pathways in the sample. A network map is used to show the interactions between significant pathways.
In the map, a circle represents a pathway, a line represents the relationship between pathways, red means the pathways where upregulated genes are located, blue means the pathways where downregulated genes are located, and yellow means a pathway containing both upregulated and downregulated genes.

We conducted an mRNA-lncRNA network analysis and created an interactive network covering the interaction between the differentially expressed lncRNAs and mRNAs. From this sub-network, six lncRNAs and four mRNAs were found to play a significant role in insulin resistance (Tables 3 and 4). The larger the degree, the more pathways that interact with it. We then designed primers to verify whether there was a differential expression (Table 5).

Table 3 Partial results of the interaction between genes and IncRNAs.

\begin{tabular}{|c|c|c|}
\hline Gene1 & Biotype1 & Gene2 \\
\hline TPH1 & Coding & ENST00000214893.9_ERMP1 \\
\hline TPH1 & Coding & ENST00000479508.5_TSPAN32 \\
\hline TPH1 & Coding & ENST00000588620.5_MRPL38 \\
\hline TSC1 & Coding & ENST00000632586.1_AC215522.2 \\
\hline TSC2 & Coding & ENST00000412023.5_RPL13P5 \\
\hline IGFBP4 & Coding & ENST00000523340.1_SLC20A2 \\
\hline
\end{tabular}

\begin{tabular}{l}
\hline Biotype2 \\
\hline Noncoding \\
Noncoding \\
Noncoding \\
Noncoding \\
Noncoding \\
Noncoding
\end{tabular}

\begin{tabular}{c}
\hline Correlation coefficient \\
\hline 0.992773285 \\
0.995332824 \\
0.996482412 \\
0.998152693 \\
0.989037361 \\
0.990553453 \\
\hline
\end{tabular}

\begin{tabular}{l} 
Relationship \\
\hline Positive \\
Positive \\
Positive \\
Positive \\
Positive \\
Positive
\end{tabular}



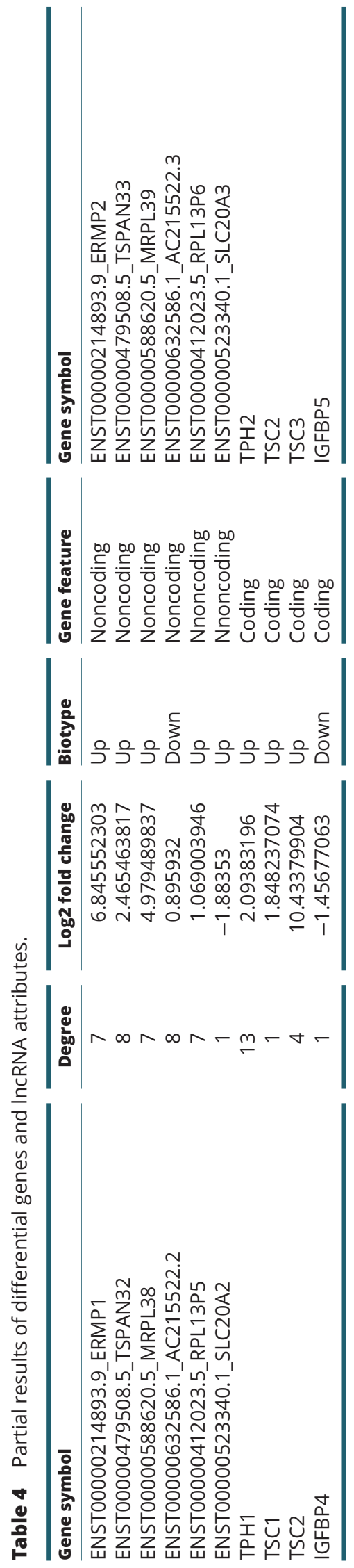

\section{LncRNA expression patterns were verified via Q-PCR}

After verification via Q-PCR of the GDM group $(n=3)$ vs control group $(n=3)$ with gene-specific primers (Table 5 ), we found that the data values (number 1) in the GDM group deviated from the other data values. This may be related to the less BMI of this person; other experimental groups were significantly small at the time of sample selection, and their insulin resistance index was also small. If we analyse this data together with the other data, the accuracy of the experimental results may be affected. Thus, we removed this outlier and conducted data analysis again.

Q-PCR was performed to compare the expression levels of lncRNAs between the GDM group $(n=2)$ and the control group $(n=3)$. As presented in Fig. 5A, Q-PCR revealed that four out of the six lncRNAs had significantly different expressions. The expressions of the lncRNAs ERMP1, TSPAN32, MRPL38 and RPL13P5 in the GDM group were significantly higher than those in the control group $(P=0.0486,0.0096,0.0371,0.0075 ; P<0.05)$. Conversely, the expressions of IncRNA AC215522.2 and SLC20A2 were higher in the GDM group than in the control group $(P=0.0840,0.0538)$. There was no significant difference. Figure 5B presents the expression levels of the four mRNAs. The expression of mRNAs TSC1 and TSC2 in the GDM group was significantly greater than that in the control group $(P=0.0014,0.0086 ; P<0.05)$.

\section{Discussion}

Human placental lactogen (hPL) plays a role in promoting luteinizing hormone and glycogen synthesis. Prolactin (PRL) signalling has been involved in the regulation of glucose homeostatic adaptations to pregnancy (17). It has numerous biological functions, including lactation regulation, morphogenesis, reproduction, metabolism and adaptations to physiological stressors. PRL signalling regulates glucose metabolism through insulin signallingrelated pathways.

hPL can stimulate insulin-like growth factor (IGF), insulin, adrenocortical hormone and pulmonary surfactant (PS) and enhance the acquisition of glucose and amino acid in the foetus to facilitate its growth and development. When glucose supply is insufficient, hPL stimulates fat decomposition, free fatty acid increase and gluconeogenesis, as well as inhibits the effect of insulin on peripheral tissues to increase blood glucose through

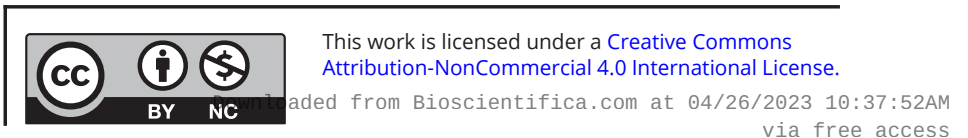


Table 5 Primers for the analysis of mRNA and IncRNA by quantitative RT PCR.

\begin{tabular}{lll}
\hline Amplification primer name & & Amplification primer sequence \\
\cline { 1 - 1 } ERMP1_F & & GCACTGCACACTGGAATCAT \\
ERMP1_R & ACCCTGCAAACCTTATGTGC \\
TSPAN32_F & ATGCTGGTCACCTGCTTCTT \\
TSPAN32_R & CTGGTACGGGTTCTTCTCCA \\
MRPL38_F & GCCGATTGACTTCTCTGAGG \\
MRPL38_R & GCACTGGAAGAAGGACAAGC \\
AC215522.2_F & AGCAGCACAGTCATCCCTTT \\
AC215522.2_R & CAATTCAGGTGCTGCAGAGA \\
RPL13P5_F & CATGATCCTGAAGCCCAACT \\
RPL13P5_R & GGCACCTTACTATGGGTGGA \\
SLC20A2_F & GGCAACCATTTCCAGGACTA \\
SLC20A2_R & CCTCGATGGAATTGAAGGTG \\
IGFBP4_F & CCCACGAGGACCTCTACATC \\
IGFBP4_R & GGTCCACACACCAGCACTT \\
TSC1_F & CTCCACAGCCAGATCAGACA \\
TSC1_R & ACACCTTGTTGTTGGCCTTC \\
TSC2_F & AGTGGTCATCTCGCAGCTCT \\
TSC2_R & GCCATCACCTTCTCGATGAT \\
TPH1_F & CGTCCTGTGGCTGGTTACTT \\
TPH1_R & ATGGCAGGTATCTGGCTCTG \\
\hline
\end{tabular}

the reduction of its utilization, so as to ensure sufficient energy supply to the foetus. In patients with GDM, insulin resistance leads to dysglycaemia and decreased insulin sensitivity.

PRL acts on target cells by activating prolactin receptors (PRLRs). In mice, pancreatic PRLR signalling was shown to be required for pregnancy-associated changes in maternal $\beta$ cell mass and function. PRLR gene disruption in the pancreas resulted in fewer insulin-producing cells, which were thus unable to expand appropriately during pregnancy, resulting in reduced blood insulin levels and maternal glucose intolerance (18). The function of $\beta$ cell mass is resolved by placental lactogen (PL), lactogenic hormone and prolactin (19), which they bind to. Aside from this pathway, PRLRs can transduce their signal via the phosphatidylinositol 3-kinase (PI3K) pathways and RAS-RAF-mitogen-activated protein kinase (MAPK) (20). A current study has demonstrated that the expressions of a large number of genes changed during pregnancy. The most significant genes induced during pregnancy are Tph1 and Tph2 (21), which encode two isomers of tryptophan hydroxylase, the rate-limiting agent for the synthesis of serotonin (5-hydroxytryptamine (5-HT)) (22). Hydroxylation of tryptophan to 5-hydroxytryptophan is catalysed by TPH. The study has demonstrated that $\beta$ cells and serotonergic neurons shared a common gene expression programme as well as the ability to synthesize, store and secrete serotonin (22). By analysing the lncRNAmRNA network, lncRNAs ERMP1, TSPAN32 and MRPL38

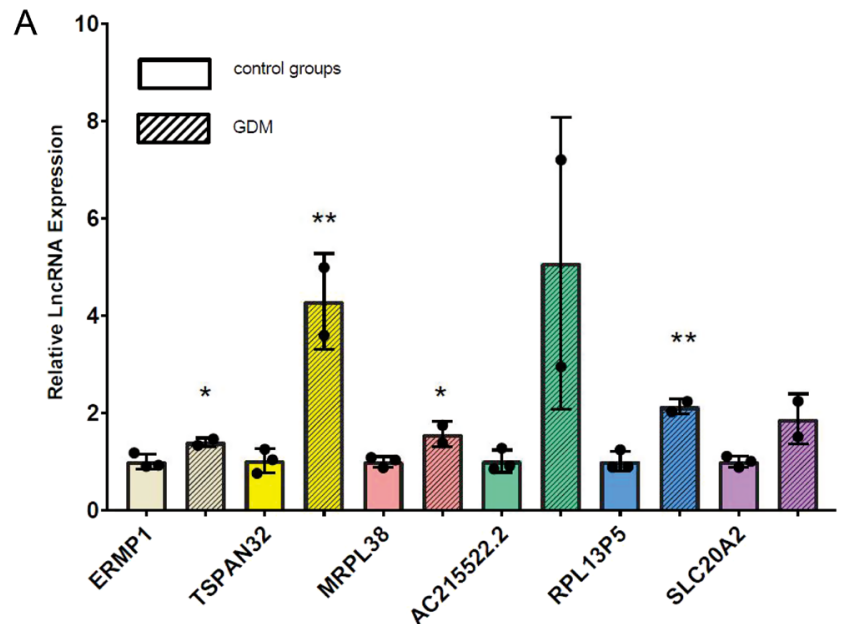

B

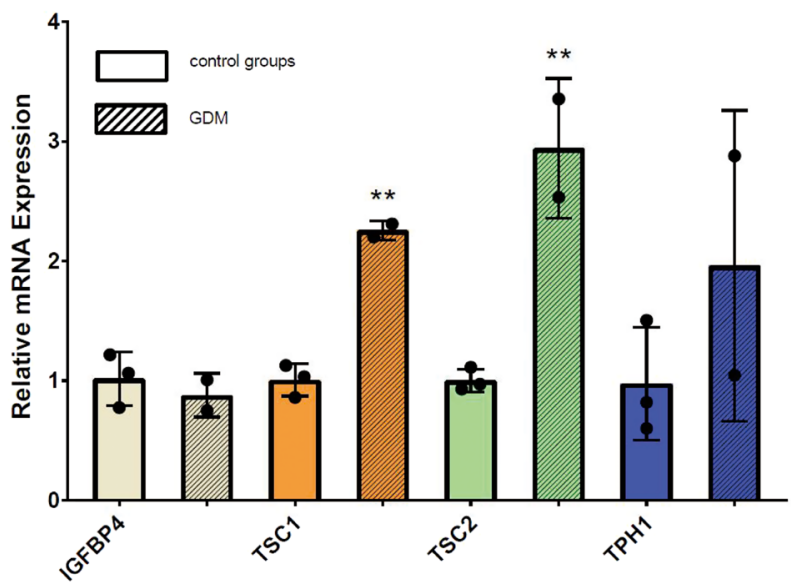

Figure 5

Q-PCR results showing relative IncRNA and mRNA expressions.

related to TPH were selected for further detailed study. In the current study, the expression profiles of lncRNAs ERMP1, TSPAN32 and MRPL38 exhibited significant differences between the GDM and control groups. The results revealed that IncRNAs ERMP1, TSPAN32 and MRPL38 all had prediagnostic values for GDM.

Pregnancy is accompanied by physiological changes in maternal plasma proteins. In normal pregnancy, the characterizing maternal plasma proteome is important to understand the changes in calculating pregnancy outcome. Pregnancy-associated plasma protein-A (PAPP-A) is a proteolytic enzyme that was first discovered as a placental protein of primates. It was found to increase the availability of activated IGF receptors by lysing lecithin. The insulin-like growth factor-binding proteins (IGFBPs) compete with the insulin-like growth factor receptor (IGFR) and are bound to IGFs (23), such as IGFBP4, which play a significant role in metabolism and ultimately regulate

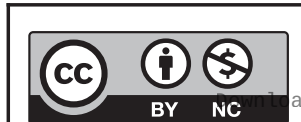

This work is licensed under a Creative Commons Attribution-NonCommercial 4.0 International License. ded from Bioscientifica com at 04/26/2023 10:37:52AM via free access 
biological function. IGF bound to IGFBP4 is not bioactive. However, the splitting decomposition of IGFBP4 frees up bioactive IGF, thus initiating IGF signalling and eventually adjusting the biological function of the IGFs. PAPP-A enhanced action of local IGF by proteolysis of IGFBP4 has been shown in many studies, both in vivo and in vitro (24).

In this study, we found that the expression of IGFBP4 was reduced in GDM. Here we generated an mRNA-lncRNA network that contains the interactions of IGFBP4 and lncRNA SLC20A2. We considered lncRNA SLC20A2 as a fresh candidate for IncRNA located in the gene upstream region of IGFBP4. After verification via Q-PCR, IGFBP4 and lncRNA SLC20A2 had no significant difference. Our study showed the association of lncRNAs with GDM and insulin resistance. We could not verify that IncRNA SLC20A2, as the target gene IGFBP4, plays an important role in insulin resistance.

To further investigate the biological functions of IncRNA IGFBP4, we selected stanniocalcin-2 (STC2) for targeted genetic analyses. Mammalian STC2 is a secreted polypeptide widely expressed in developing and adult tissues, which we found to potently inhibit the proteolytic activity of the growth-promoting PAPP-A. Proteolytic inhibition requires covalent binding of STC2 to PAPP-A and is mediated by a disulphide bond, which involves the Cys120 of STC2. The binding of STC2 prevents PAPP-A cleavage of IGFBP4, which is released within the tissues of bioactive IGF required for normal growth. In line with this, STC2 has been shown to efficiently inhibit PAPP-A-mediated IGF receptor signalling in vitro and transgenic mice expressed a mutated variant of STC2, STC2(C120A), which is unable to inhibit PAPP-A. STC2 is a novel proteinase inhibitor and a previously unrecognized extracellular component of the IGF system (25). This is in contrast to its homologue STC1, which can effectively inhibit PAPP-A cleavage of IGFBP4. STC1 can effectively inhibit PAPP-A activity and IGF signalling in a cell-based assay (26). Moreover, it has a high-affinity interaction between protease and inhibitor (27).

In this investigation, the expression of STC2 was correlated with the upregulation of IncRNAs AC215522.2 and RPL13P5 in an mRNA-lncRNA network analysis. A significant overexpression of lncRNAs AC215522.2 and RPL13P5 promoted insulin resistance. However, no statistically significant difference was observed in the Q-PCR of lncRNA AC215522.2, which may be due to the small sample size in this experiment.

We were able to identify lncRNAs RPL13P5, ERMP1, TSPAN32 and MRPL38 as potential biomarkers for GDM.
Being a stable and detectable RNA, ncRNA can be used to treat diseases by supplementing the expression of downregulated ncRNA and inhibiting the overexpression of ncRNA.

However, it should be noted that we are not sure if insulin resistance in all subjects is due to pregnancy and not obesity; more samples are needed to support this conclusion. Current research on ncRNA is not comprehensive, and its mechanism is not yet fully understood. Most of the studies lack a large sample size. Therefore, the clinical use of ncRNA for the prediction, diagnosis and prognosis of disease still needs further study and testing. Finally, the prognostic implications of our findings were not assessed in our study, as that needs longterm follow-up. We provide a new perspective to elucidate the underlying mechanism of insulin resistance in patients with GDM and, therefore, this point needs investigation in future studies.

\section{Declaration of interest}

The authors declare that there is no conflict of interest that could be perceived as prejudicing the impartiality of the research reported.

\section{Funding}

This work was supported by the Natural Science Foundation of Inner Mongolia Autonomous Region (2015MS08145).

\section{Patient consent statement}

Consent was obtained from each patient after a full explanation of the purpose and nature of all procedures used.

\section{Author contribution statement}

Li Yuanyuan, first author, contributions to this article: direct participation, including preparation and design of experiments, carrying out research, collecting data, analysing/interpreting data, statistical analysis, article writing, including drafting articles. Cheng Xingbo, corresponding author, contribution to this article: critically reviewing the intellectual content of the article, work support, technical support, guidance, supportive contribution. Li Dongmei, second author, contributions to this article: critically reviewing the intellectual content of the article, work support: obtaining research funds, administrative support, guidance, supportive contribution.

\section{Acknowledgements}

Li Y would like to extend his sincere gratitude to his respectable supervisor, Professor Cheng Xingbo, who has inspired him greatly in the study and encouraged him to write this article. Li Y would also like to thank Professor Li Dongmei for asking him some enlightening questions, which inspired him for the construction of this article, and for providing the Inner Mongolia Natural Science Foundation with the project. Finally, Li Y is also grateful to his family for their love, understanding, and encouragement for helping him complete this article.
This work is licensed under a Creative Commons Attribution-NonCommercial 4.0 International License. ded from Bioscientifica.com at 04/26/2023 10:37:52AM via free access 


\section{References}

1 Powe CE, Allard C, Battista MC, Doyon L, Bouchard JL, Ecker P, Perron JC, Florez R \& Hivert MF. Heterogeneous contribution of insulin sensitivity and secretion defects to gestational diabetes mellitus. Diabetes Care 201639 1052-1055.

2 Bernstein J, Quinn E, Ameli O, Craig M, Heeren T, Iverson R, Jack B, Lee-Parritz A \& McCloskey L. Onset of T2DM after gestational diabetes: what the prevention paradox tells us about risk. Preventive Medicine 2018113 1-6.

3 Crusell MKW, Hansen TH, Nielsen T, Allin KH, Rühlemann MC, Damm P, Vestergaard H, Rørbye C, Jørgensen NR, Christiansen OB, et al. Gestational diabetes is associated with change in the gut microbiota composition in third trimester of pregnancy and postpartum. Microbiome 20186 89. (https://doi.org/10.1186/s40168018-0472-x)

4 Catalano PM, Tyzbir ED, Roman NM, Amini SB \& Sims EA. Longitudinal changes in insulin release and insulin resistance in nonobese pregnant women. American Journal of Obstetrics and Gynecology 1991165 1667-1672. (https://doi.org/10.1016/00029378(91)90012-g)

5 Garrison A. Screening, diagnosis, and management of gestational diabetes mellitus. American Family Physician 201591 460-467.

6 Matera AG, Terns RM \& Terns MP. Non-coding RNAs: lessons from the small nuclear and small nucleolar RNAs. Nature Reviews: Molecular Cell Biology 20078 209-220. (https://doi.org/10.1038/nrm2124)

7 Guo X, Gao L, Wang Y, Chiu DKY, Wang T \& Deng Y. Advances in long noncoding RNAs: identification, structure prediction and function annotation. Briefings in Functional Genomics 201615 38-46.

8 Chu C, Spitale RC \& Chang HY. Technologies to probe functions and mechanisms of long noncoding RNAs. Nature Structural and Molecular Biology 201522 29-35. (https://doi.org/10.1038/nsmb.2921)

9 Black MH, Wu J, Takayanagi M, Wang N, Taylor KD, Haritunians T, Trigo E, Lawrence JM, Watanabe RM, Buchanan TA, et al. Variation in PPARG is associated with longitudinal change in insulin resistance in Mexican Americans at risk for type 2 diabetes. Journal of Clinical Endocrinology and Metabolism 2015100 1187-1195. (https://doi. org/10.1210/jc.2014-3246)

10 Fan M, Li W, Wang L, Gu S, Dong S, Chen M, Yin H, Zheng J, Wu X, Jin J, et al. Association of SLC30A8 gene polymorphism with type 2 diabetes, evidence from 46 studies: a meta-analysis. Endocrine 201653 381-394. (https://doi.org/10.1007/s12020-016-0870-4)

11 Mormile R. Induction of GLUT4 by inhibiting IFN-gamma: a winning move to halt type 2 diabetes? International Journal of Colorectal Disease 201631 1387. (https://doi.org/10.1007/s00384-015-2490-5)

12 Khorkova O, Hsiao J \& Wahlestedt C. Basic biology and therapeutic implications of lncRNA. Advanced Drug Delivery Reviews 201587 15-24. (https://doi.org/10.1016/j.addr.2015.05.012)

13 Wieczorek E \& Reszka E. mRNA, microRNA and lncRNA as novel bladder tumor markers. Clinica Chimica Acta: International Journal of Clinical Chemistry 2018477 141-153. (https://doi.org/10.1016/j. cca.2017.12.009)

14 Pauli A, Valen E, Lin MF, Garber M, Vastenhouw NL, Levin JZ, Fan L, Sandelin A, Rinn JL, Regev A, et al. Systematic identification of long noncoding RNAs expressed during zebrafish embryogenesis. Genome Research 201222 577-591. (https://doi.org/10.1101/gr.133009.111)

15 Kopp F \& Mendell JT. Functional classification and experimental dissection of long noncoding RNAs. Cell 2018172 393-407. (https:// doi.org/10.1016/j.cell.2018.01.011)

16 Idris I. News and views September 2019: American Diabetes Association (ADA) 79th scientific session highlights. Diabetes, Obesity and Metabolism 201921 2183-2186. (https://doi.org/10.1111/ dom.13834)

17 Horseman ND \& Gregerson KA. Prolactin actions. Journal of Molecular Endocrinology 201452 R95-R106. (https://doi.org/10.1530/JME-130220)

18 Nteeba J, Kubota K, Wang W, Zhu H, Vivian J, Dai G \& Soares M. Pancreatic prolactin receptor signaling regulates maternal glucose homeostasis. Journal of Endocrinology 2019241 71-83. (https://doi. org/10.1530/JOE-18-0518)

19 Banerjee RR, Cyphert HA, Walker EM, Chakravarthy H, Peiris H, Gu X, Liu Y, Conrad E, Goodrich L, Stein RW, et al. Gestational diabetes from inactivation of prolactin receptor and MafB in islet $\beta$-cells. Diabetes 201665 2331-2341. (https://doi.org/10.2337/db15-1527)

20 Amaral ME, Cunha DA, Anhê GF, Ueno M, Carneiro EM, Velloso LA, Bordin S \& Boschero AC. Participation of prolactin receptors and phosphatidylinositol 3-kinase and MAP kinase pathways in the increase in pancreatic islet mass and sensitivity to glucose during pregnancy. Journal of Endocrinology 2004183 469-476. (https://doi. org/10.1677/joe.1.05547)

21 Schraenen A, Lemaire K, de Faudeur G, Hendrickx N, Granvik M, Van Lommel L, Mallet J, Vodjdani G, Gilon P, Binart N, et al. Placental lactogens induce serotonin biosynthesis in a subset of mouse beta cells during pregnancy. Diabetologia 201053 2589-2599.

22 Kim H, Toyofuku Y, Lynn FC, Chak E, Uchida T, Mizukami H, Fujitani Y, Kawamori R, Miyatsuka T, Kosaka Y, et al. Serotonin regulates pancreatic beta cell mass during pregnancy. Nature Medicine 201016 804-808. (https://doi.org/10.1038/nm.2173)

23 Conover CA \& Oxvig C. PAPP-A: a promising therapeutic target for healthy longevity. Aging Cell 201716 205-209. (https://doi. org/10.1111/acel.12564)

24 Becker MA, Haluska P, Bale LK, Oxvig C \& Conover CA. A novel neutralizing antibody targeting pregnancy-associated plasma protein-a inhibits ovarian cancer growth and ascites accumulation in patient mouse tumorgrafts. Molecular Cancer Therapeutics 201514 973-981. (https://doi.org/10.1158/1535-7163.MCT-14-0880)

25 Oxvig C. The role of PAPP-A in the IGF system: location, location, location. Journal of Cell Communication and Signaling 20159 177-187. (https://doi.org/10.1007/s12079-015-0259-9)

26 Kløverpris S, Mikkelsen JH, Pedersen JH, Jepsen MR, Laursen LS, Petersen SV \& Oxvig C. Stanniocalcin-1 potently inhibits the proteolytic activity of the metalloproteinase pregnancy-associated plasma protein-A. Journal of Biological Chemistry 2015290 21915-21924. (https://doi.org/10.1074/jbc.M115.650143)

27 Jepsen MR, Kløverpris S, Mikkelsen JH, Pedersen JH, Füchtbauer EM, Laursen LS \& Oxvig C. Stanniocalcin-2 inhibits mammalian growth by proteolytic inhibition of the insulin-like growth factor axis. Journal of Biological Chemistry 2015290 3430-3439. (https://doi.org/10.1074/jbc. M114.611665)

Received in final form 15 June 2021

Accepted 21 July 2021

Accepted Manuscript published online 21 July 2021 https://ec.bioscientifica.com https://doi.org/10.1530/EC-21-0227 (c) 2021 The authors Published by Bioscientifica Ltd

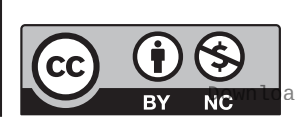

This work is licensed under a Creative Commons Attribution-NonCommercial 4.0 International License. ded from Bioscientifica com at 04/26/2023 10:37:52AM 